\title{
Photon-excited collective modes in a surface alloy
}

\author{
S. R. Barman \\ Inter University Consortium, Khandwa Road, Indore, 452001, M.P., India \\ and Fritz-Haber Institut der Max-Planck-Gesellschaft, 14195 Berlin, Germany \\ C. Stampfl \\ Fritz-Haber Institut der Max-Planck-Gesellschaft, 14195 Berlin, Germany \\ P. Häberle \\ Departmento de Física, Universidad Técnica Federico Santa María, Valparaiso, Chile \\ K. Horn \\ Fritz-Haber Institut der Max-Planck-Gesellschaft, 14195 Berlin, Germany
}

(Received 21 June 1999)

\begin{abstract}
We investigate the influence of modifications of the surface charge-density profile on photon-excited collective excitations of valence electrons, through a comparison of the angle- and energy-resolved photoyield of clean $\mathrm{Al}$ and the $\mathrm{Al} / \mathrm{Na}$ "surface alloy." Although the charge-density profile is considerably different for the clean and surface alloy systems, the Al multipole plasmon energy is essentially similar, in contrast to what one might expect from simple charge-density arguments. A Na multipole plasmon is observed only when excess $\mathrm{Na}$ is deposited over the surface alloy. We base a qualitative explanation for these observations on results of surface electronic structure calculations using total-energy density-functional theory.
\end{abstract}

The study of the dynamic response of electrons at the surface of simple metals and thin films to the influence of an externally applied electromagnetic field provides an ideal testing ground for an understanding of the interaction of the field with fluctuating charges at the surface, and a theoretical description of many-body effects in solids. Large variations in the field at the surface in a specific regime around $80 \%$ of the bulk plasmon frequency $\hbar \omega_{p}$ were predicted by Feibelman, ${ }^{1}$ and established experimentally through photoyield measurements by Levinson, Plummer, and Feibelman $^{2}$ for $\mathrm{Al}(100)$. This enhancement, which is caused by the $\nabla \cdot A$ term in the photoemission matrix element $M_{\text {if }}$ $=\left\langle\phi_{f}|A \cdot \nabla+\nabla \cdot A| \phi_{i}\right\rangle$, was later found to be connected with a specific surface collective excitation, the multipole plasmon, ${ }^{3}$ identified in electron-energy-loss spectra of $\mathrm{Na}$ and $\mathrm{K}$ films by Tsuei et ll $^{4}$ The multipole mode was predicted in studies of surface excitations using hydrodynamic theory by Bennett as early as $1970 .{ }^{3}$ Subsequent theoretical investigations using the time-dependent local-density approximation (TD-LDA) and LDA-based random phase approximation calculations were successful in explaining the experimental observations of surface collective excitations from clean surfaces and thin films of simple, free-electronlike metals. ${ }^{5-10}$

Here we report on the influence of complex surface charge-density profiles on the energy and intensity of collective modes, especially the multipole plasmon. We investigate the substitutional $\mathrm{Al}(100)-c(2 \times 2)$ : Na surface alloy, where every second $\mathrm{Al}$ atom in the top layer is displaced by a $\mathrm{Na}$ atom. While the ground-state electronic structure of surfaces has been the subject of hundreds of investigations, both experimental and theoretical, the optical response of a surface alloy phase, with its strong modification of the surface charge density, has not been studied in the literature to the best of our knowledge. Our paper demonstrates that, contrary to what one might expect on the basis of a simple model for the "dilution" of the electron gas at the surface, collective excitations, which are thought to depend on the chargedensity profile and the density of the electron gas at the surface, are not affected by the "surface alloying" process. These surprising findings are qualitatively explained on the basis of the surface electronic structure calculated by totalenergy density-functional theory using the local density approximation (DFT-LDA).

The measurements were carried out at the 1 meter SeyaNamioka beamline at the BESSY (Berliner ElektronenSpeicherring-Gesellschaft für Synchrotronstrahlung) storage ring using a commercial angle-resolving electron spectrometer (ADES400 from Vacuum Generators, UK) at a base pressure of $6 \times 10^{-11}$ mbar. A prepolished Al crystal was cleaned by repeated sputtering and heating cycles. Na was deposited using SAES getter sources with the substrate at room temperature. The growth was monitored by changes in substrate work function and low-energy electron diffraction. The angle- and energy-resolved photoyield (AERPY) spectra were measured by recording the intensity near the Fermi level $\left(E_{F}\right)$ as a function of photon energy in the normal emission with $p$-polarized light incident at $45^{\circ}$. The advantage of the AERPY technique over the total yield measurement is that it does not make a contribution to the photoemission intensity from the inelastically scattered secondary photoelectrons, which is difficult to analyze and may also depend on surface quality and sample preparation history. In order to decrease the measuring time and improve the quality of the data, measurements were mostly performed in the constant initial state (CIS) mode at binding energies of $0.3 \mathrm{eV}$ 


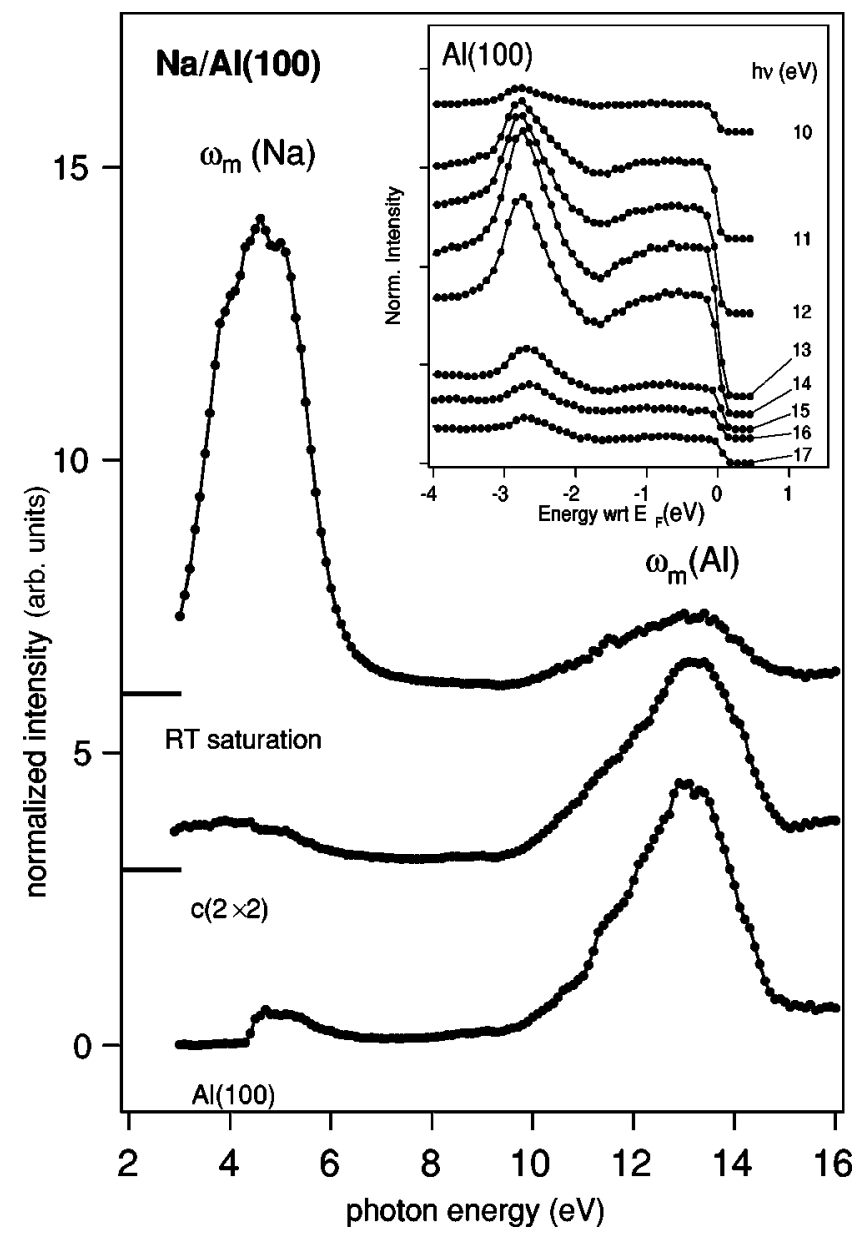

FIG. 1. Photoyield (AERPY) spectra for clean $\mathrm{Al}(100)$, the $\mathrm{Al}(100)-c(2 \times 2)$ : Na surface alloy phase, and a saturation monolayer coverage of $\mathrm{Na}$ on the surface alloy at room temperature. The spectra are shifted with respect to each other, and the zero of each spectrum is indicated on the left vertical axis. $\omega_{m}(\mathrm{Al})$ and $\omega_{m}(\mathrm{Na})$ correspond to $\mathrm{Al}$ and $\mathrm{Na}$ multipole frequencies, respectively. The inset shows a set of normal emission photoemission spectra for $\mathrm{Al}(100)$, recorded at different photon energies in the multipole plasmon region, shifted with respect to each other for clarity.

below $E_{F} \cdot{ }^{11,12}$ Energy distribution curves (EDC's) were also recorded. The data were normalized in the same way for all spectra, by measuring the photon flux using a gold mesh and a GaAsP diode. Corrections were made for higher-order light and analyzer transmission function.

The inset of Fig. 1 shows a representative set of normal emission EDC spectra from clean $\mathrm{Al}(100)$ for a small range of photon energies near the maximum of photoemission enhancement (which occurs around $13 \mathrm{eV}$ ) normalized to the photon flux. These data show that there is a rather uniform intensity enhancement of the entire spectrum, including the region near $E_{F}$ and the surface-state emission at $2.7 \mathrm{eV}$ below $E_{F}$. The intensity near $E_{F}$, recorded in the CIS mode, is shown for clean $\mathrm{Al}(100)$ as well as for different overlayers in Fig. 1. The enhancement of photoemission intensity around $13 \mathrm{eV}$ is due to the large variation of the surface electromagnetic field, i.e., the $\boldsymbol{\nabla} \cdot A$ term in the photoemission matrix element; this corresponds to the multipole plasmon of the clean Al surface. The peak position of $13 \mathrm{eV}$ is similar to that of $\mathrm{Al}(111),{ }^{12}$ and is in general agreement with theoreti-

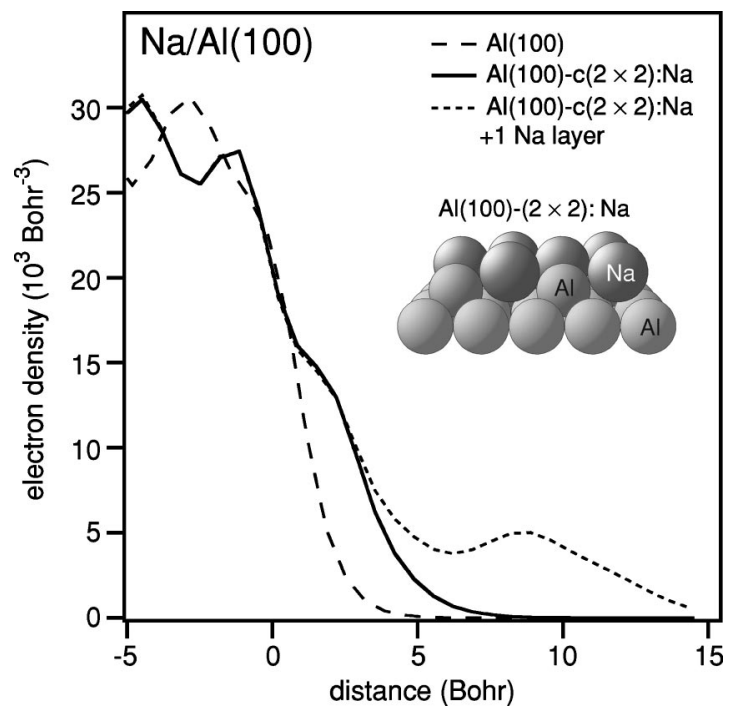

FIG. 2. Variation of the laterally averaged electron density perpendicular to the surface, calculated using $a b$ initio total energy DFT-LDA for clean $\mathrm{Al}(100)$, the $\mathrm{Al}(100)-c(2 \times 2)$ : Na surface alloy phase, and a saturation monolayer coverage of $\mathrm{Na}$ on the surface alloy. The profiles are aligned at the falling edge of the Al substrate at $20 \times 10^{-3}$ bohr $^{-3}$, where the zero of the horizontal axis is defined. The geometry of the surface alloy is shown as an inset.

cal predictions based on TD-LDA calculations using jellium. ${ }^{13}$ There is also an overall agreement with the experimental data of Levinson, Plummer, and Feibelman, ${ }^{2}$ although the peak is located at about $12.5 \mathrm{eV}$ in that work.

The structure of the $\mathrm{Al}(100)-c(2 \times 2): \mathrm{Na}$ surface alloy phase is sketched in the inset of Fig. 2. The corresponding AERPY spectrum shows the multipole peak to occur at exactly the same energy as on the clean surface, (center trace) with a slightly lower intensity. Subsequent deposition of $\mathrm{Na}$ at room temperature leads to an extinction of the $c(2 \times 2)$ overlayer LEED spots, and the corresponding AERPY spectrum for a saturation Na coverage on top of the surface alloy phase shows that the $\mathrm{Al}$ multipole peak at $13 \mathrm{eV}$ is largely suppressed, while a broad and intense feature at around 4.6 $\mathrm{eV}$, in the region of $\mathrm{Na}$ collective excitation, emerges. The work-function cutoff in $\mathrm{Al}(100)$ at $4.5 \mathrm{eV}$ shifts to lower energies with $\mathrm{Na}$ deposition because of $\mathrm{Na}$-induced work function lowering.

TD-LDA calculations for smooth alkali-metal overlayers, performed both by means of jellium slabs and threedimensional realistic atomic layers, have predicted the presence of multipole and standing-wave-like bulk-plasmon excitation in these overlayers, the latter being caused by the presence of the vacuum/adlayer and adlayer/substrate interfacial barrier. ${ }^{6,8}$ In these studies it was found that collective excitations in overlayers depend sensitively on the shape of the charge-density distribution. We have therefore performed self-consistent $a b$ initio total-energy DFT-LDA calculations of the ground-state charge-density profiles for the three layers, for which results are shown in Fig. 1; the details of the calculation are described elsewhere. ${ }^{14}$ These profiles are shown in Fig. 2. The clean surface has the charge "spillout" commonly found in simple metal surfaces. The surface alloy phase has a narrow step at about $55 \%$ of the maximum charge density, which is due to the fact that every second $\mathrm{Al}$ 
atom is replaced by a $\mathrm{Na}$ atom in this phase; the step is thus dominated by contributions from $\mathrm{Al}$ atoms. The ground-state electronic structure of this phase is characterized by a coupling between the $\mathrm{Na}$ and $\mathrm{Al}(100)$ surface bands. There is a charge transfer from the region on top of the $\mathrm{Na}$ atoms to that of $\mathrm{Al}$, and the charge-density plot along the surface shows that there is a large lateral corrugation. ${ }^{15}$ In fact, transfer of the $\mathrm{Na}$ valence electron is almost complete, and hence the $\mathrm{Na}$-derived band is unoccupied, while the surface-state band derived from the $\mathrm{Al}$ vacancy structure is fully occupied, with a "band gap" of $3.5 \mathrm{eV}$ across $E_{F}$. This was verified through angle-resolved photoemission measurements, ${ }^{15}$ which also show that the $\mathrm{Al}$ surface band into which the $\mathrm{Na}$ electrons are transferred has a higher effective mass $\left(m^{*}\right.$ $\left.=1.55 m_{e l}\right)$ compared to clean $\operatorname{Al}(100)\left(1.18 m_{e l}\right)$. Thus the response of the $\mathrm{Al}(100)-c(2 \times 2): \mathrm{Na}$ phase must be dominated by the $\mathrm{Al}$ atom, since basically its structure is that of highly corrugated open $\mathrm{Al}$ surface. This indeed appears to be the case, since the shape of the response in the $10-15-\mathrm{eV}$ region is very similar to that of clean $\mathrm{Al}$, as shown by the AERPY data in Fig. 1.

In previous investigations of the response of alkali-metal overlayers to an incident electromagnetic field, it was found that the emergence of collective excitations was connected with the formation of a wide and well-defined plateau in the surface charge density, such that the substrate-overlayer and overlayer-vacuum interfaces are clearly separated. .,8,10,11 $^{\text {The }}$ energy of the collective excitations is determined by the charge density of the plateau. This requirement for $\hbar \omega_{m}$ to appear can in fact be seen directly from a comparison of our data for the Na saturation coverage (top trace of Fig. 1) with the charge density calculated for two $\mathrm{Na}$ atoms per unit cell (which corresponds to one extra layer of coverage) in Fig. 2. The latter has a well-developed plateau, which apparently gives rise to the peak in the AERPY spectrum centered at 4.6 $\mathrm{eV}$, associated with the $\mathrm{Na}$ multipole plasmon. The peak position is in good agreement with the multipole plasmon observed on two smooth Na layers on $\mathrm{Al}^{11,16}$ However, in contrast to the smooth Na layers, in this case the multipole and bulklike plasmon modes are not clearly separated, although the total width of the response $(2 \mathrm{eV})$ is similar. This is probably due to presence of the large lateral chargedensity corrugations in the substrate-overlayer interface, as shown recently for Li overlayers. ${ }^{11}$ In the experiment, an increase in the $\mathrm{Na}$ coverage beyond the $c(2 \times 2)$ alloy leads to a gradual increase of the Na multipole feature, and a concomitant attenuation of the $\mathrm{Al}$ multipole peak. That the $\mathrm{Na}$ multipole feature appears in the AERPY spectrum for coverages beyond the surface alloy phase is plausible, since this layer has an occupied $\mathrm{Na}$ band as found in our DFT-LDA calculations.

Since jellium slab model calculations work well for smooth alkali-metal overlayers, ${ }^{8}$ a simple Drude model relating the surface charge density to the plasmon modes, according to $\hbar \omega_{p}=\sqrt{4 \pi n e^{2} / m}$, applies for these overlayers. If the same reasoning is applied for a 55\% reduction in density at the step in the charge density profile (Fig. 2), one might expect a mode to appear at lower energy (around $9 \mathrm{eV}$ ) for the alloy surface, since that the multipole plasmon generally occurs around $0.8 \hbar \omega_{p} .{ }^{17}$ However, our experimental results (center trace in Fig. 1) show that there is no distinct collec- tive excitation in this region; only the $\mathrm{Al}$ multipole plasmon is apparent, and a slight enhancement in the low energy region which is related to the so-called threshold excitation. ${ }^{8}$ Thus the narrow steplike deviation from the clean $\mathrm{Al}$ chargedensity profile in $\mathrm{Al}(100)-c(2 \times 2): \mathrm{Na}$ is not wide and diffuse enough to sustain a separate collective mode. We observe that only when an extra $\mathrm{Na}$ layer is formed on the alloy surface, are the collective modes observed. With the formation of the Na layer the surface is no longer an alloy, and the previous theoretical results ${ }^{8}$ for smooth alkali-metal overlayers apply to an interpretation of this situation.

Our conclusions concerning the influence of the shape of surface charge distribution on the collective modes in $\mathrm{Al}(100)-c(2 \times 2): \mathrm{Na}$ are supported by similar observations for a more complex surface alloy phase, the $\mathrm{Al}(111)-(2$ $\times 2$ ): Na phase, which consists of a four-layer Na-Al-Na-Al sandwich. Again, no change in the Al multipole plasmon is found in this system either, and only weak features appear in the $\mathrm{Na}$ collective excitation region, which grow into intense features corresponding to the Na multipole and bulk-plasmon excitations when going to room temperature saturation coverage.$^{18}$ In fact, previous DFT-LDA calculations for this system show that in this case there is also a charge transfer from $\mathrm{Na}$ to $\mathrm{Al}$ with a filled $\mathrm{Al}$ band and an unoccupied $\mathrm{Na}$ band. ${ }^{15,14}$ The similarity of the ground-state electronic structure explains the similarity of these two systems in the photoresponse.

The above discussions provide a qualitative interpretation of our experimental observations of the behavior of collective excitation for the $\mathrm{Na} / \mathrm{Al}$ alloy surface on the basis of rigorous DFT-LDA calculations of the ground state. A more quantitative picture should emerge from response calculations for this system. Ishida and Liebsch modeled the response of the $\mathrm{Al}(100)-c(2 \times 2): \mathrm{Na}$ surface alloy in the $\mathrm{Na}$ plasmon energy region using the TD-LDA. ${ }^{19}$ They used a four-layer slab on a jellium substrate for calculations of the response in the $\mathrm{Na}$ energy region. They calculated the ground-state electronic structure by a self-consistent Green's-function method with experimental structural parameters, and the dynamic response was calculated within the TD-LDA. ${ }^{17}$ The imaginary part of the centroid of the induced charge density, which is proportional to the total yield, was compared with the experiment. Their results showed a small enhancement around $5 \mathrm{eV}$, which is in fair agreement with our data. However, in their calculation of the response in the $\mathrm{Al}(100)-c(2 \times 2): \mathrm{Na}$, they used a much simplified model with only one $c(2 \times 2)$-reconstructed $\mathrm{Al}$ layer on a jellium substrate, and no $\mathrm{Na}$ atoms, i.e., vacancies replacing the $\mathrm{Na}$ at the substitutional sites. Their data for such a geometry show an overall shift of the Al multipole plasmon peak toward lower energy to $10.5 \mathrm{eV}$, in stark contrast with our experimental data. They also observed an increase of the $\mathrm{Al}$ multipole peak intensity in the substitutional phase by about a factor of 4 compared to the clean surface, which is not seen in our experimental data either. A reason for this discrepancy could be the use of the simplified model; for example, due to the omission of $\mathrm{Na}$ atoms in the calculation ${ }^{19}$ the polarization of the surface is probably overestimated. Our DFT-LDA calculations show that there are considerable differences between the ground-state electronic structure of the $c(2 \times 2)-\mathrm{Al}$ and the $\mathrm{Al}(100)-c(2 \times 2): \mathrm{Na}$ 
vacancy; in particular, the effect of the almost complete charge transfer from $\mathrm{Na}$ band to the $\mathrm{Al}$ band stabilizes the $\mathrm{Al}$ band. This effect, which dominates the alloy electronic structure, was not taken into account in the calculation of Ishida and Liebsch. ${ }^{19}$ Thus the results indicate that an accurate description of the ground-state electronic structure is necessary to describe the collective excitations on surface alloys, even though the simplified models work well for the constituent free-electron-like pure metals, e.g., $\mathrm{Na}$ and $\mathrm{Al}$, which constitute the surface alloy. ${ }^{17}$ In addition, for computational reasons the substrate surface region in which the dynamical response was calculated in Ref. 19 had to be kept rather small $\left[(10-20) a_{0}\right]$. While this is adequate at low frequencies in the region of the alkali-metal excitations, it is too narrow near the Al surface multipole frequency. From jellium calculations it is $\operatorname{known}^{17}$ that the fluctating density associated with this mode extends rather far into the solid $\left[(50-100) a_{0}\right]$.
In summary, although the charge density profile of clean $\mathrm{Al}$ is considerably altered by surface alloying with $\mathrm{Na}$, the $\mathrm{Al}$ multipole energy remains essentially unchanged. This is qualitatively explained by DFT-LDA-based ground-state calculations which show that there is an almost complete charge transfer of the $\mathrm{Na}$ valence electron to $\mathrm{Al}$ in the substitutional layer. The step in the calculated charge-density profile is too narrow to sustain a separate collective excitation. Na-related collective modes are observed only when one $\mathrm{Na}$ layer is deposited over the susbstitutional layer which forms a wide plateau in the density profile.

We thank A. Liebsch for useful discussions, and L. Aballe and C. Chen for help during measurements. This work was supported by a European Community Grant No. CI1* CT93-0059 (DG 12 HSMU), Fondecyt Chile Grant No. 1970122, the Bundesministerium für Bildung und Forschung under Grant No. 056220LA3, and DST-DLR Project No. IND 99/038 .
${ }^{1}$ P. J. Feibelman, Phys. Rev. Lett. 34, 1092 (1975).

${ }^{2}$ H. Levinson, E. W. Plummer, and P. J. Feibelman, Phys. Rev. Lett. 43, 952 (1979).

${ }^{3}$ A. J. Bennett, Phys. Rev. B 1, 203 (1970).

${ }^{4}$ K.-D. Tsuei et al., Phys. Rev. Lett. 64, 44 (1990).

${ }^{5}$ A. Liebsch, Phys. Rev. B 36, 7378 (1987).

${ }^{6}$ A. Liebsch, Phys. Rev. Lett. 67, 2858 (1991).

${ }^{7}$ A. Liebsch, G. Benemanskaya, and M. Lapusklin, Surf. Sci. 302, 303 (1994).

${ }^{8}$ H. Ishida and A. Liebsch, Phys. Rev. B 45, 6171 (1992).

${ }^{9}$ P. J. Feibelman, Prog. Surf. Sci. 12, 287 (1982).

${ }^{10}$ J. E. Inglesfield and E. Wikborg, J. Phys. F: Met. Phys. 5, 1706 (1975).

${ }^{11}$ S. R. Barman et al., Phys. Rev. B 57, 6662 (1998).
${ }^{12}$ S. R. Barman, P. Häberle, and K. Horn, Phys. Rev. B 58, R4285 (1998).

${ }^{13} \hbar \omega_{m}$ from total photoyield calculations is $12 \mathrm{eV}$ (Refs. 5 and 9), while from emitted total photoyield, which is more appropriate for comparison with present data $\hbar \omega_{m}$, is $13.4 \mathrm{eV}$ (Ref. 7).

${ }^{14}$ C. Stampfl and M. Scheffler, Surf. Sci. 319, L23 (1994).

${ }^{15}$ C. Stampfl et al., Phys. Rev. B 57, 15251 (1998).

${ }^{16} \mathrm{~S}$. R. Barman et al. (unpublished).

${ }^{17}$ A. Liebsch, Electronic Excitations at Metal Surfaces (Plenum, New York, 1997).

${ }^{18}$ S. R. Barman and K. Horn, Appl. Phys. A: Solids Surf. 69, 519 (1999).

${ }^{19}$ H. Ishida and A. Liebsch, Phys. Rev. B 57, 12558 (1998). 